\title{
Energy efficient walking with central pattern generators: from passive dynamic walking to biologically inspired control
}

\author{
B. W. Verdaasdonk - H. F. J. M. Koopman • \\ F. C. T. van der Helm
}

Received: 26 December 2008 / Accepted: 27 April 2009 / Published online: 6 June 2009

(C) The Author(s) 2009. This article is published with open access at Springerlink.com

\begin{abstract}
Like human walking, passive dynamic walking - i.e. walking down a slope with no actuation except gravity - is energy efficient by exploiting the natural dynamics. In the animal world, neural oscillators termed central pattern generators (CPGs) provide the basic rhythm for muscular activity in locomotion. We present a CPG model, which automatically tunes into the resonance frequency of the passive dynamics of a bipedal walker, i.e. the CPG model exhibits resonance tuning behavior. Each leg is coupled to its own CPG, controlling the hip moment of force. Resonance tuning above the endogenous frequency of the $\mathrm{CPG}-$ i.e. the CPG's eigenfrequency - is achieved by feedback of both limb angles to their corresponding CPG, while integration of the limb angles provides resonance tuning at and below the endogenous frequency of the CPG. Feedback of the angular velocity of both limbs to their corresponding CPG compensates for the time delay in the loop coupling each limb to its CPG. The resonance tuning behavior of the CPG model allows the gait velocity to be controlled by a single parameter, while retaining the energy efficiency of passive dynamic walking.
\end{abstract}

B. W. Verdaasdonk $(\varangle) \cdot$ H. F. J. M. Koopman Department of Bio-mechanical Engineering, Faculty of Engineering Technology, University of Twente, P.O. Box 217, 7500 AE, Enschede, The Netherlands e-mail: bart.verdaasdonk@bosch.com

H. F. J. M. Koopman

e-mail: h.f.j.m.koopman@utwente.nl

F. C. T. van der Helm

Department of BioMechanical Engineering,

Faculty of Mechanical Engineering, Delft University of Technology, Mekelweg 2, 2628 CD, Delft, The Netherlands

e-mail: f.c.t.vanderhelm@tudelft.nl
Keywords Legged locomotion - Central pattern generator · Resonance tuning - Energy efficiency · Passive dynamic walking $\cdot$ Route to chaos

\section{Introduction}

Walking is an important function of the human movement apparatus. Healthy people are not aware of the complexity of walking; they walk with little effort, without consciously thinking about it. During the stance phase, the stance leg acts as an inverted pendulum with a mass on top. Therefore, human walking is statically unstable. Thus, an interesting question is how 'human gait' can be dynamically stable, robust and energy efficient at the same time. In this study, we focus on the energy efficiency of walking.

Observations of human walking show that most of the muscles are only highly active at the beginning and end of the stance and swing phase (Inman et al. 1981). Ballistic gait models are based on these observations. They exploit their natural dynamics: the legs behave as a jointed pendulum, moving passively through the swing phase under the action of gravity (Mochon and McMahon 1980). Therefore, ballistic gait models are very energy efficient. However, the lack of control during the swing phase gives them poor robustness against perturbations. Even small perturbations can accumulate during the swing phase into large foot placement errors at heel strike. Hence, control is necessary during the entire gait cycle in order to obtain robust gait, but should leave the natural dynamics intact as much as possible in order to obtain energy efficient gait. The latter is not the mainstream starting-point for bipedal gait control, since path-following control and control based on keeping the COP (center of pressure) within the foot base of support are common practice in robotics. However, this kind of control will force the actual 
walking cycle to differ from the natural walking cycle. This costs a lot of energy and is therefore unlikely to be used in human gait. Neural oscillators in the spinal cord, termed central pattern generators (CPGs), are likely to play a key role in providing energy efficient human gait, for the following reasons: firstly, many animals use CPGs to control gait. CPGs excite the muscles in a periodic fashion, giving rise to stable locomotion. Most evidence of the existence of CPGs in vertebrates comes from lamprey (e.g. Grillner et al. 1981), rats (e.g. Cazalets et al. 1995; Sqalli-Houssaini et al. 1993), and cats (e.g. Amemiya and Yamaguchi 1984; Shik et al. 1966); for reviews see MacKay-Lyons (2002), Whelan (1996), and Grillener et al. (1998). Secondly, it has been shown that a simple model of a CPG is able to provide energy efficient rhythmic single limb movement (Verdaasdonk et al. 2006, 2007b). Although no direct evidence of CPGs in the human body has been found yet, indications of their existence are present (e.g. see Dimitrijevic et al. 1998).

It has been shown in bipedal gait models (Taga 1995a;Taga et al. 1991; Verdaasdonk et al. 2004b, 2007a) that mutual entrainment of the CPGs with the musculo-skeletal system and its environment creates a stable limit cycle which is quite robust against perturbations (Taga 1995b; Verdaasdonk et al. 2007a). The robustness is caused by the coupling between CPGs and musculo-skeletal system, which continuously cause perturbed state variables to be pulled back towards the limit cycle during the entire gait cycle.

The energy efficiency of CPG controlled gait has not been considered up to now. In previous studies (Verdaasdonk et al. 2006, 2007b), it was shown that CPGs are very suitable for energy efficient and robust rythmic single limb movement. The CPGs entrain to reflex inputs, such as muscle length and velocity. This means that the CPGs adapt their outputs (i.e. frequency and amplitude) to the 'mechanical oscillator' (i.e. the arm or leg) in such a way that stable rhythmic movement is obtained, which is robust and energy efficient. The energy efficiency is obtained by tuning into the resonance frequency of the musculo-skeletal system. The type of afferent feedback to the flexor and extensor centers of the CPG plays a crucial role in obtaining this resonance tuning behavior of the CPG. In this study, the 'mechanical oscillator' is a simple gait model and we investigate whether the principles of CPG-controlled energy efficient and robust rhythmic limb movement shown in single limb movement (Verdaasdonk et al. 2006, 2007b) also apply to walking.

Passive dynamic walking (McGeer 1990)—i.e. walking down a slope with no actuation except gravity-is an extreme form of ballistic walking (Mochon and McMahon 1980) and is very energy efficient. Drawbacks of passive dynamic walking are poor robustness, as shown by the small size of its basin of attraction (Schwab and Wisse 2001), and of course the lack of controllability. The goal of this study is to control the quality of bipedal gait in terms of stride length, stride

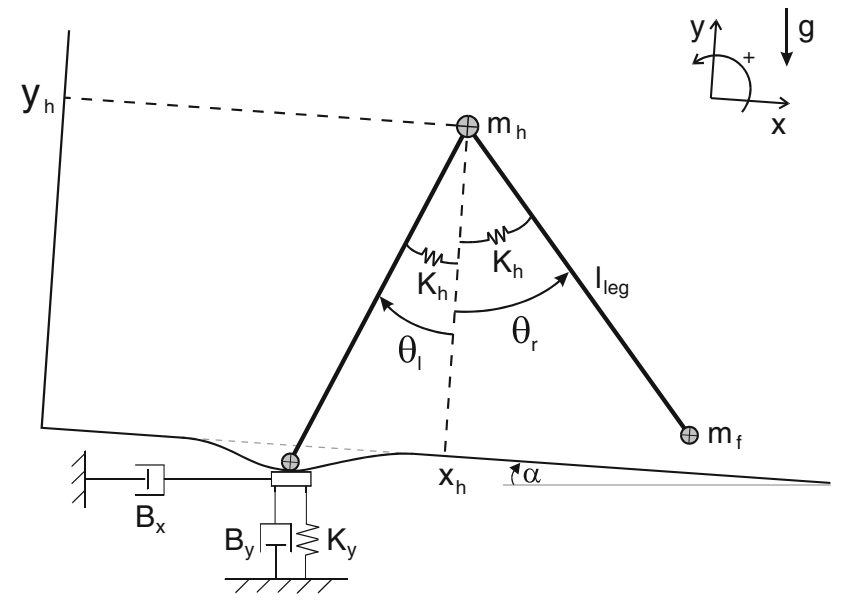

Fig. 1 The passive dynamic walker. The model has a large hip mass $m_{\mathrm{h}}$ relative to the mass of the feet $m_{\mathrm{f}}$. The legs have length $l_{\text {leg }}$ and the hips have rotational stiffness $K_{\mathrm{h}}$. The model is able to walk passively down a slope $\alpha$, for which gravity $g$ supplies all the energy needed to overcome impact losses. Foot-ground contact is modeled by damping $B_{\mathrm{x}}$ in the $x$-direction and by damping $B_{\mathrm{y}}$ and stiffness $K_{\mathrm{y}}$ in the $y$-direction. The generalized co-ordinates are the hip angles $\theta_{\mathrm{r}}$ and $\theta_{\mathrm{l}}$ and the co-ordinates of the hip mass $x_{\mathrm{h}}$ and $y_{\mathrm{h}}$

period and thus velocity, while keeping the energy efficient behavior of passive dynamic walking intact. To achieve this goal, we start off with a passive dynamic walking model and subsequently add biologically inspired control. This control consists of a CPG tightly coupled to each hip joint.

\section{Methods}

In order to investigate if CPGs are able to maintain the energy efficiency of passive dynamic walkers, we start off with a passive dynamic walking model (Sect. 2.1). Subsequently, each leg is locally coupled to its own CPG at the hip joint (Sect. 2.2). This coupling is afferent and efferent, associated with sensory feedback and motor control, respectively. The total bipedal walker can be seen as two coupled oscillators. One oscillator is the passive dynamic walker (Fig. 1) and the other oscillator is the neural oscillator, which consists of one CPG per hip joint (Fig. 2). By coupling these two oscillators tightly, the combined system will oscillate at one frequency and result in stable gait. The energy efficiency of the CPGcontrolled gait model is discussed in Sect. 2.3 and depends on two factors. The first is the ability of the neural oscillator to tune into the resonance frequency of the passive dynamic walker, which is termed resonance tuning ability. The second is the energy losses by damping, mainly caused by impact during heel strike.

\subsection{The passive dynamic walker}

The passive dynamic walking model consists of two rigid legs (Fig. 1). The legs are connected at the hip by a frictionless 


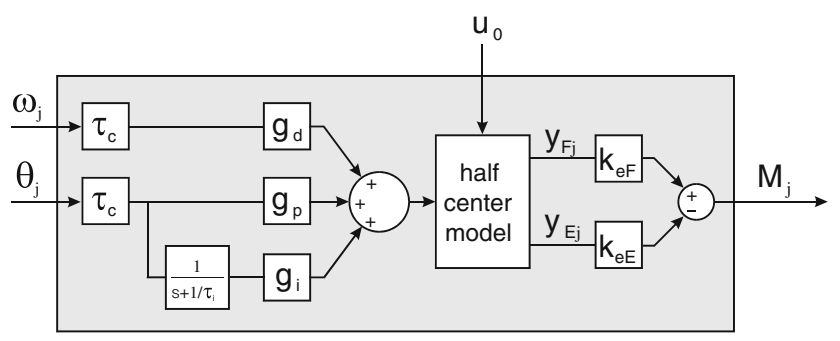

Fig. 2 The CPG model for leg $j(j=\{r, l\})$. It has supra-spinal input $u_{0}$ and afferent input of leg angle $\theta_{\mathrm{j}}$ and angular velocity $\omega_{\mathrm{j}}$, which is fed to the half-center model with gains $g_{\mathrm{p}}$ and $g_{\mathrm{d}}$ and time delay $\tau_{\mathrm{c}}$. The leg angle is integrated by a leaking integrator with large time constant $\tau_{\mathrm{i}}$ and is fed to the half-center model with gain $g_{\mathrm{i}}$. The half-center model is the basic rhythm generator, which outputs $y_{\mathrm{Fj}}$ and $y_{\mathrm{Ej}}$ are coupled to the limb by efferent gains $k_{\mathrm{eF}}$ and $k_{\mathrm{eE}}$ to provide a hip moment of force $M_{\mathrm{j}}$ from the torso

hinge joint and have point feet. The model only has three point masses: one point mass $m_{\mathrm{h}}$ at the hip and two smaller point masses $m_{\mathrm{f}}$ located at the feet. Since the walker does not have knees, ground-clearance during swing phase is provided artificially ("Appendix A"). The passive dynamic walker is able to walk passively down a small slope $\alpha$ (i.e. positive $\alpha$ means declination). In that case, gravity provides exactly as much energy during the gait cycle as is lost by damping (mainly at heel strike). The stride frequency of this walkerits 'resonance frequency'-is changed by adding rotational hip stiffness $K_{\mathrm{h}}$.

The model without hip stiffness (i.e. $K_{\mathrm{h}}=0$ ) resembles minimalistic models of passive dynamic walking, such as the 'simplest walking model' (Garcia et al. 1998) and the 'compass gait model' (Goswami et al. 1997). The major difference between our model and most other passive dynamic walking models (Borzova and Hurmuzlu 2004; Garcia et al. 1998; Goswami et al. 1997; McGeer 1990; van der Linde 1999) is the way that foot-ground contact is described. The ground reaction force is modeled by viscous damping in both $x$ - and $y$-direction and stiffness only in $y$-direction (see "Appendix A" for details). This makes investigation of walking on different types of ground (e.g. a slippery surface) possible, although this was not performed in this study. Most other models make use of impact equations at heel strike, after which the legs are switched and a new swing phase starts (e.g. Schwab and Wisse 2001). Our model is a continuoustime model, able to walk 'forever' instead of one step at a time. We used the approach of Lagrange in order to construct the equations of motion (see "Appendix B"). The equations of motion are expressed in terms of four generalized co-ordi-

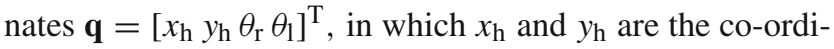
nates of the hip mass $m_{\mathrm{h}}$ parallel and perpendicular to ground level, respectively, and $\theta_{\mathrm{r}}$ and $\theta_{\mathrm{l}}$ are the right and left leg angles, relative to the perpendicular of the ground.

\subsection{Coupling the legs to central pattern generators}

Central patterns generators (CPGs) are neural networks in the spinal cord, which output periodic excitations to the muscles, even in a completely isolated spinal cord (Nishimaru and Kudo 2000; Sqalli-Houssaini et al. 1993). The CPG model used in this study is similar to the 'PID-type' CPG, discussed in Verdaasdonk et al. (2006). The CPG model features Positional, Integral and Derivative feedback of the limb angle to the flexor and extensor centers of the CPG. Previous studies (Verdaasdonk et al. 2006, 2007b) have shown that this type of afferent feedback is crucial in providing energy efficient control in rhythmic single limb movement. In these studies, feedback of positional information was shown to provide resonance tuning above the CPG's endogenous frequency $f_{\mathrm{CPG}}$ (i.e. the natural frequency at which the uncoupled CPG oscillates). Integral feedback was shown to provide resonance tuning at and below $f_{\mathrm{CPG}}$. Feedback of velocity information (i.e. derivative feedback) was shown to be necessary in order to compensate for the time delay in the loop coupling limb to the CPG. Resonance tuning is not possible at high movement frequencies without it (Verdaasdonk et al. 2006).

The CPG model is shown in Fig. 2 and its equations are summarized in "Appendix C". The basic rhythm generator of the CPG is a half-center model. It is based on the work of Matsuoka $(1985,1987)$ and was discussed at length in Verdaasdonk et al. (2006). The literature (Barbeau et al. 1999; Burke 2001; McCrea 2001; Van de Crommert et al. 1998; Whelan 1996) suggests feedback from Ia and II fibers to the flexor and extensor centers are present during walking. This is abstracted in our model as delayed feedback of the leg's angle $\theta_{\mathrm{j}}$ and angular velocity $\omega_{\mathrm{j}}(j=\{r, l\})$ to the half-center model with gains $g_{\mathrm{p}}$ and $g_{\mathrm{d}}$ and time delay $\tau_{\mathrm{c}}$. An internal process of the CPG 'integrates' the delayed leg angle $\theta_{\mathrm{j}}\left(t-\tau_{\mathrm{c}}\right)$ by a leaking integrator with large time constant $\tau_{\mathrm{i}}$, which is subsequently fed to the half-center model with gain $g_{\mathrm{i}}$. The hip joint of each leg $j$ is locally coupled to its own CPG.

Our passive dynamic walker has no trunk, which has little effect on the motion of the legs (McGeer 1990). However, in order to still be able to control the hips separately, as humans do, a virtual torso is introduced: the torso is considered perpendicular to the ground level and is fixed. The output of the flexor center $y_{\mathrm{F}}$ and extensor center $y_{\mathrm{E}}$ are amplified by the respective efferent gains $k_{\mathrm{eF}}$ and $k_{\mathrm{eE}}$ to form the motor signals to the muscles that provide hip flexion and extension. The 'muscles' are modeled most simply, translating the motor signals linearly to a hip moment of force $M_{\mathrm{j}}$ relative to the torso $(j=\{r, l\})$. Supra-spinal input $u_{0}$ to the CPG can be changed to increase or decrease the outputs of the flexor and extensor center, and thus the hip moments of force.

It is important to note that the afferent feedback gains are not chosen by trial and error, but by analyzing the properties 
needed by the afferent feedback to assure resonance tuning. This analysis is discussed thoroughly in Verdaasdonk et al. (2006) and will not be repeated here. In summary, it comes down to choosing the pole of the PI feedback to the CPG's flexor and extensor centers at the endogenous frequency of the CPG ( $g_{\mathrm{i}}=2 \pi f_{\mathrm{CPG}} g_{\mathrm{p}}$, see "Appendix C") and choosing the zero of the PD feedback according to the experienced time delay $\left(g_{\mathrm{d}}=0.08 g_{\mathrm{p}}\right.$ for $50 \mathrm{~ms}$ time delay, see "Appendix C"). This way, the only real variable of the afferent feedback is its strength $g_{\mathrm{p}}$, which can be used to vary the gait velocity (see Sect. 3.3).

\subsection{Energy efficiency analysis}

In engineering, the energy efficiency of a system is often defined as the ratio between the amount of total performed work (i.e. the sum of positive and negative work) and the energy expenditure needed to achieve this work. The efficiency $\eta$ of the CPG-controlled actuation at the hips can be determined during one gait cycle:

$\eta=100 \frac{W}{E_{\exp }} \%$

with $W$ the total amount of work performed by the hip muscles during one gait cycle and $E_{\exp }$ the accompanying energy expenditure. The work $W$ is necessary to compensate for the damping losses, which mainly consist of impact losses that occur when the heel strikes the ground at the end of the swing phase. The hip muscles of our gait model do not store energy, which means that performing positive as well as negative work costs energy. Thus, the energy expenditure $E_{\text {exp }}$ is calculated as follows:

$E_{\exp }=\sum_{\mathrm{j}}\left(\int_{0}^{T}\left|\left(M_{\mathrm{j}} \omega_{\mathrm{j}}\right)\right| \mathrm{d} t\right) \quad \mathrm{J} \quad j=\{r, l\}$

with $E_{\text {exp }}$ the energy expended during one gait cycle, $T$ the period of the gait cycle, $M_{\mathrm{j}}$ the muscle moment of force about hip joint $j$ relative to the torso, and $\omega_{\mathrm{j}}$ the angular velocity of leg $j$. When the signs of $M_{\mathrm{j}}$ and $\omega_{\mathrm{j}}$ are equal, positive work is done; else negative work is done. The energy efficiency ratio $\eta$ is $100 \%$ if only positive work is performed by the hip muscles during the gait cycle. Note that for perfect resonance tuning in a mass-spring system with spring extension $x$ and sinusoidal actuation force $F$, the energy efficiency ratio $\eta$ is $100 \%$. This is due to the fact that the phase of the transfer function $H(s)=x(s) / F(s)$ is $-90^{\circ}$ at the resonance frequency, i.e. the force $F$ and velocity $v=\mathrm{d} x / \mathrm{d} t$ are in phase and only positive work is performed. In a similar way, an energy efficiency ratio $\eta$ of $100 \%$ is obtained for our CPG-controlled gait model if the stride frequency equals the 'resonance frequency' of the passive dynamic walker. Resonance tuning leaves the natural exchange between potential and kinetic energy intact: no active braking of the legs is necessary and thus only positive work is performed by the hip muscles. Forcing the gait model to walk slower or faster than its natural frequency at given gait velocity, would imply a control that actively brakes and thus performs negative work.

The efficiency ratio $\eta$ is a good measure of the resonance tuning capability of the CPGs. However, it gives an incomplete view of the energy efficiency of walking. Even if the efficiency $\eta$ of the hip muscles is $100 \%$, a given distance can be walked with different energy expenditures. For our simple gait model, this is caused by different collision losses at heel strike (i.e. the negative work done by the dampers $B_{\mathrm{x}}$ and especially $B_{\mathrm{y}}$ ) for different gait qualities (i.e. different stride length $S$ and/or stride period $T$ ). Therefore, the energy expenditure per unit distance walked $E_{\mathrm{m}}$ is also determined (normalized to mass). In the field of bio-mechanics this is a widely used measure for the energy efficiency of walking (Koopman et al. 1989; McMahon 1984). The energy $E_{\exp }$ expended during one gait cycle is divided by the stride length $S$ and total mass $\left(2 m_{\mathrm{f}}+m_{\mathrm{h}}\right)$ of the gait model in order to yield $E_{\mathrm{m}}$ :

$E_{\mathrm{m}}=\frac{E_{\exp }}{S\left(2 m_{\mathrm{f}}+m_{\mathrm{h}}\right)} \mathrm{J} /(\mathrm{kg} \mathrm{m})$

To perform the aforementioned energy efficiency analysis, periodic cycles of the gait model have to be found and analyzed. This is done with help of Poincaré mapping as discussed in depth in Verdaasdonk et al. (2007a)

\section{Results}

\subsection{Passive dynamic walking}

The gait model (Fig. 1) is able to walk down certain slopes $\alpha$ without any control, i.e. it is a passive dynamic walker (Sect. 2.1). For small slopes $\alpha$, the gravitational energy received by the passive dynamic walker-i.e. the work $W_{\mathrm{g}}$ performed by gravity - is proportional to the slope. To be precise, it equals $\left(2 m_{\mathrm{f}}+m_{\mathrm{h}}\right) g \sin (\alpha)$ Joule per meter walked. Figure 3 shows the stride length $S$, stride period $T$ and gait velocity $v$ against downward slope $\alpha$ for all stable gait solutions. Since the stride period $T$ is almost invariant with respect to the slope, which was also shown by Kuo (2002) and Garcia et al. (1998), increasing gait velocity $v=S / T$ implies increasing stride length $S$. A larger stride length means more mechanical work has to be done by gravity in order to redirect the hip mass velocity at heel strike (Donelan et al. 2002), which corresponds to a steeper slope in passive dynamic walking. Hence, the gait model walks faster down a steep slope than a shallow one. 


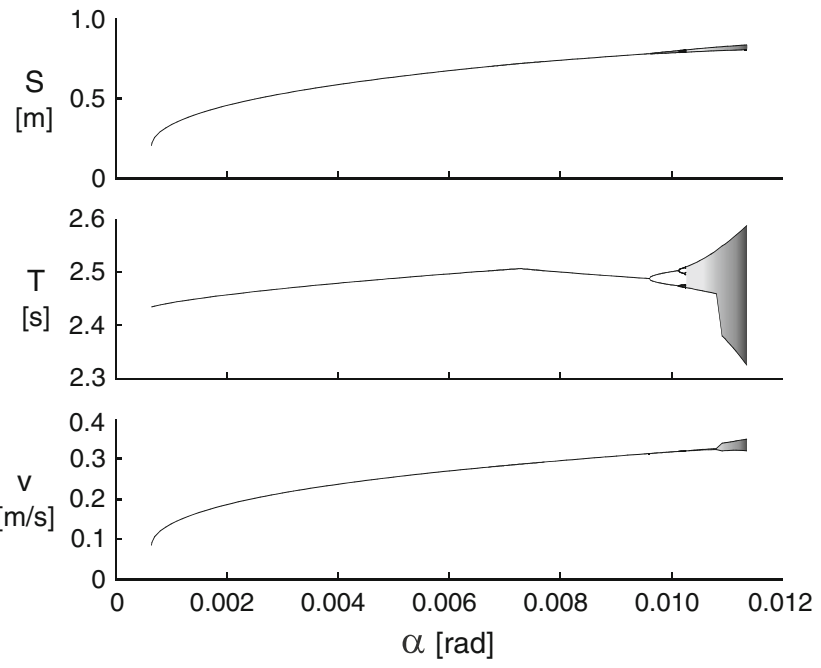

Fig. 3 Passive dynamic walking for increasing slope $\alpha$. The top graph shows the stride length $S$, the middle graph shows the stride period $T$, and the bottom graph shows the gait velocity $v$. At steep slopes there is a route to chaos, which is depicted in Fig. 4. Beyond slopes of $0.0114 \mathrm{rad}$ stable walking is not possible

Stable passive dynamic walking is not possible for slopes below $6.44 \times 10^{-4} \mathrm{rad}$, because in our model the foot of the stance leg sinks a little into the ground, which is modeled as a spring-damper. For very small stride lengths this sinking depth is relative large compared to the angle between both legs at heel strike. Therefore, after heel strike, the foot of the former stance leg does not lift above ground level and the walker stumbles and falls. For higher values of the slope the walker undergoes a series of bifurcations, which eventually lead to chaotic gait. Finally, at a slope of $0.0114 \mathrm{rad}$, the gait becomes unstable. Beyond this slope stable gait is not possible.

Figure 4 shows the route to chaos in terms of the maximal segment angle of the right leg $\theta_{\mathrm{r}}$ during one gait cycle. This route to chaos resembles the ones in Garcia et al. (1998) and Goswami et al. (1998), except that it starts of with a pitchfork bifurcation instead of a period doubling bifurcation. This is due to the different way in which gait is modeled in this study. Our model is a continuous-time model in which a complete stride (i.e. two steps) corresponds to the smallest possible recurrent period, while for the models in Garcia et al. (1998) and Goswami et al. (1998) one step is the smallest possible recurrent period, as they swap the indices of the legs after each heel strike (i.e. right becomes left and vice versa). Thus, the pitchfork bifurcation in the bifurcation diagram corresponds to the slope beyond which asymmetrical gait, i.e. a limping gait, emerges. Figure 4 shows a zoom-in up to the fourth period doubling bifurcation. The onset of chaos can be estimated with the help of Feigenbaum's universal scaling law (Feigenbaum 1978), which is shown in Eq. 4.

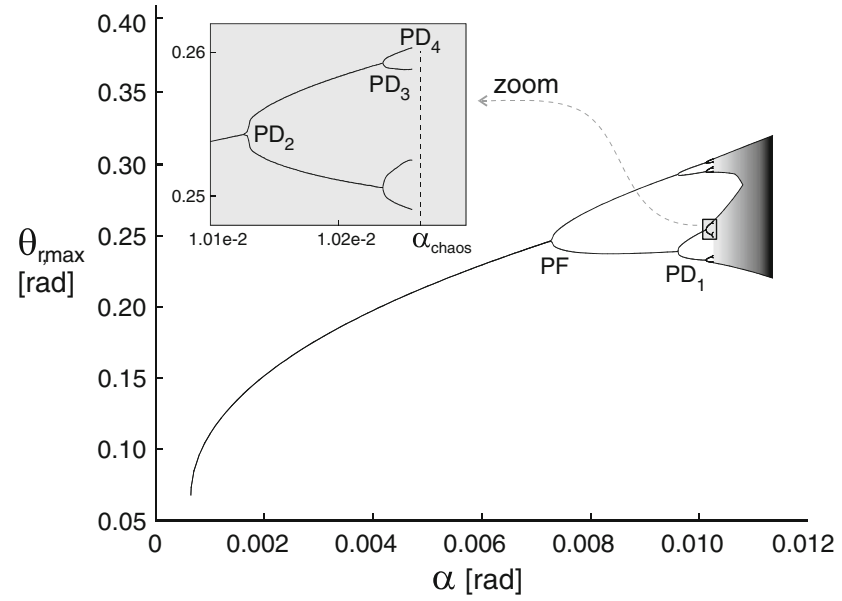

Fig. 4 Route to chaos. The passive dynamic walker initially exhibits a pitchfork bifurcation, marked $P F$, and subsequently period doubling bifurcations, marked $P D_{\mathrm{n}}$, for increasing slope $\alpha$. The zoom-in in the upper-left corner shows up to the fourth period doubling bifurcation and the estimated slope $\alpha_{\text {chaos }}$ at which chaotic gait begins (for $n \rightarrow \infty$ )

$\lim _{\mathrm{n} \rightarrow \infty} \frac{\alpha_{\mathrm{PD}, \mathrm{n}}-\alpha_{\mathrm{PD}, \mathrm{n}-1}}{\alpha_{\mathrm{PD}, \mathrm{n}+1}-\alpha_{\mathrm{PD}, \mathrm{n}}}=\delta$

where $\alpha_{\mathrm{PD}, \mathrm{n}}$ is the slope at which the $n$th period doubling bifurcation occurs and $\delta$ is the Feigenbaum constant, which has a value of $4.6692 \ldots$

From Eq. 4 the asymptotic value of the slope where infinite period doubling bifurcations have occurred can be derived. This is the slope $\alpha_{\text {chaos }}$ beyond which chaos emerges (Eq. 5).

$\alpha_{\text {chaos }}=\alpha_{\mathrm{PD}, \infty}=\frac{\alpha_{\mathrm{PD}, \mathrm{n}}-\alpha_{\mathrm{PD}, \mathrm{n}-1}}{\delta-1}$

By filling in Eq. 5 for $n=4$ a rough estimate of $\alpha_{\text {chaos }}=$ $0.0103 \mathrm{rad}$ is obtained.

Since the stride frequency of the passive dynamic walker $f_{\mathrm{PDW}}=1 / T$ is almost constant for all slopes, the walker can be thought of having a kind of 'resonance frequency'. Adding rotational hip stiffness $K_{\mathrm{h}}$ increases this resonance frequency, as is shown in Fig. 5. The stride frequency $f_{\text {PDW }}$ increases with increasing $K_{\mathrm{h}}$, while the stride length $S$ decreases. The graph shows that the gait model walks faster for a higher $K_{\mathrm{h}}$, while the energetic input from gravity remains the same $\left(\alpha=-2.6 \times 10^{-3} \mathrm{rad}\right)$. The reason for this more energy efficient gait is that energy is buffered in the hip springs, thereby decreasing the stride length and accompanying collision costs.

The resonance frequency

$f_{\text {pend }}=\frac{1}{2 \pi} \sqrt{\left(\frac{g}{l_{\text {leg }}}+\frac{K_{\mathrm{h}}}{m_{\mathrm{f}} l_{\text {leg }}^{2}}\right)}$

of the swing leg - that is, if it is swinging freely as a single hanging pendulum - is higher than the stride frequency $f_{\text {PDW }}$ (see Fig. 5), because the swing leg covers more than 

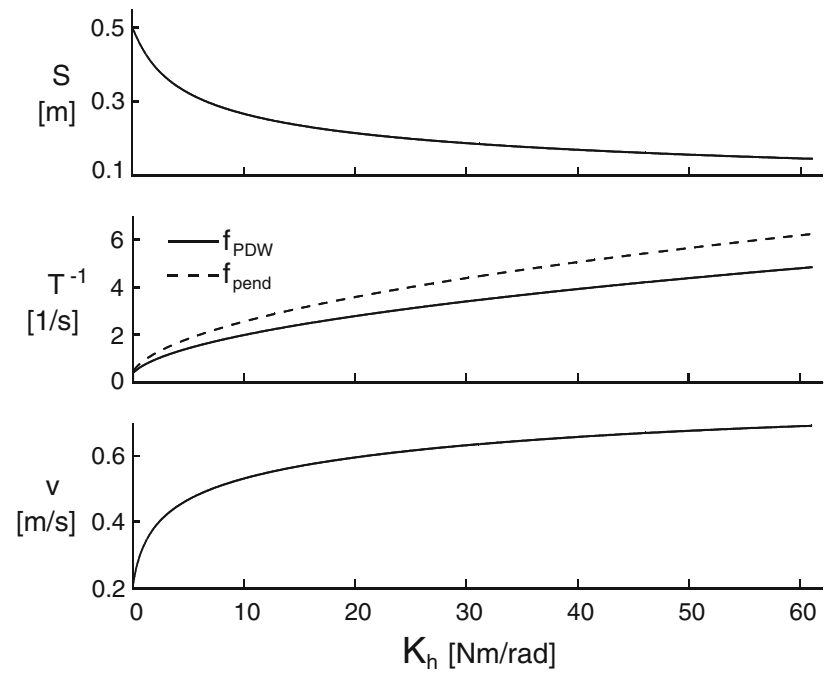

Fig. 5 Passive dynamic walking for increasing hip stiffness $K_{\mathrm{h}}$. The top graph shows the stride length $S$, the middle graph shows the stride frequency $f_{\text {PDW }}$ (solid line) versus the resonance frequency of the swing leg $f_{\text {pend }}$ as free hanging pendulum (dashed line), and the bottom graph shows the gait velocity $v$

half a period during the swing phase. The swing leg starts with a negative angular velocity to clear the ground, subsequently becomes positive to swing forward and becomes negative again just before heel strike (see Fig. 6). Actually, half a period (i.e. from zero angular velocity to zero angular velocity) of the swing phase is shorter than half a period of the free hanging pendulum. This higher 'resonance frequency' of the swing leg during gait is caused by the gravity-induced initial deceleration and subsequent acceleration of the hip mass to which the swing leg is hinged. The 'resonance frequency' of the swing leg during gait becomes closer (a) $\mathrm{K}_{\mathrm{h}}=0 \mathrm{Nm} / \mathrm{rad}$
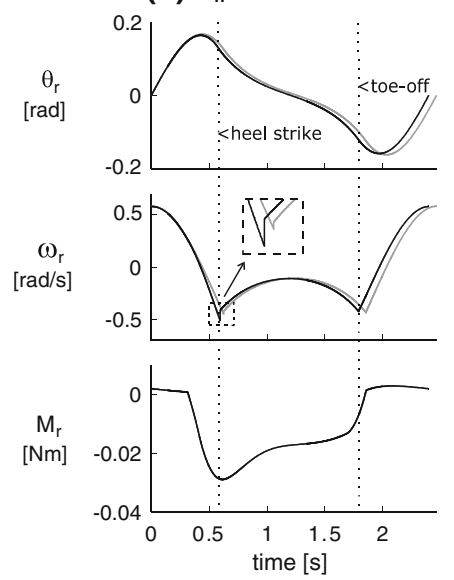

(b) $K_{\mathrm{h}}=61.05 \mathrm{Nm} / \mathrm{rad}$
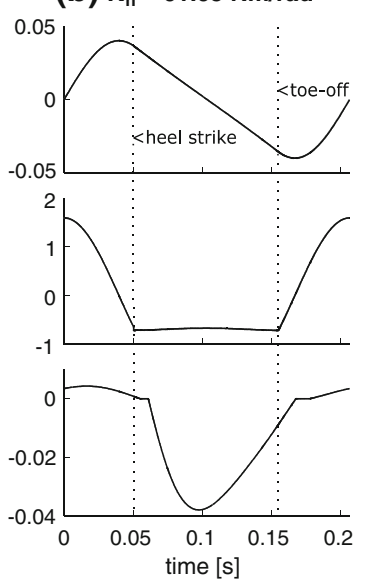

Fig. 6 Comparison of CPG-controlled walking (black lines) with passive dynamic walking (grey lines) for a no hip springs $\left(K_{\mathrm{h}}=0 \mathrm{Nm} / \mathrm{rad}\right)$ b maximal spring constant $\left(K_{\mathrm{h}}=61.05 \mathrm{Nm} / \mathrm{rad}\right)$. Time series of the angle $\theta_{\mathrm{r}}$, angular velocity $\omega_{\mathrm{r}}$ and the muscle moment of force $M_{\mathrm{r}}$ of the right leg are shown during one gait cycle to the resonance frequency of the free hanging pendulum if hip springs are added, because the exchange of energy between the hip springs and the large hip mass (i.e. the potential energy in the springs is in counter-phase with that of the hip mass) flattens the velocity profile of the stance leg (compare angular velocity $\omega_{\mathrm{r}}$ in Fig. $6 \mathrm{a}$ with that in Fig. 6b), and thereby decreases the deceleration and acceleration of the hip mass.

\subsection{Energy efficient CPG-controlled walking by resonance tuning}

\subsubsection{Comparison between CPG-controlled walking and passive dynamic walking}

In this section, the ability of the CPG to provide energy efficient gait by tuning into the resonance frequency of the passive walker is investigated. CPG-controlled walking at level ground is compared to the stable gait solutions of passive dynamic walking, shown in the previous section (see Fig. 5), for the same range of hip stiffness values $K_{\mathrm{h}}$. The strength of afferent feedback $g_{\mathrm{p}}$-and by that $g_{\mathrm{d}}$ and $g_{\mathrm{i}}$ (see "Appendix C') — is adapted for all gait solutions, in such a way that the gait velocity matches that of passive dynamic walking for all values of $K_{\mathrm{h}}$. This is done to dispose of the weak dependence of the stride frequency on gait velocity. Figure 7 compares the stride frequency $1 / T$ of CPG-controlled walking (solid line) to that of passive dynamic walking (dotted line) for the above-mentioned range of $K_{\mathrm{h}}$ values, while Fig. 8 plots the accompanying energy efficiency. The two most extreme cases are depicted in Fig. 6, which compares the time series of one gait cycle of CPG-controlled walking (black lines) with passive dynamic walking (grey lines) in case of no hip stiffness (Fig. 6a) and maximal hip stiffness (Fig. 6b). Below we discuss the results in detail.

Figure 7 shows the entrained stride frequency $1 / T$ to equal the frequency of passive dynamic walking $f_{\text {PDW }}$ very well. However, for stride frequencies below the endogenous frequency $f_{\mathrm{CPG}}$-i.e. the eigenfrequency of the CPG, which is $0.62 \mathrm{1} / \mathrm{s}$ for the parameter settings in this study-there are slight deviations (best visible in Fig. 7 by looking at $S$, as $v=S / T$ is constant for given $\left.f_{\mathrm{PDW}}\right)$. The top graph of Fig. 8 shows that this slight deviation from perfect resonance tuning at low stride frequencies causes the energy expenditure $E_{\mathrm{m}}$ of the CPG-controlled muscles (black solid line) to be somewhat higher than the work $W_{\mathrm{m}}$ (grey solid line) that they perform. This is expressed by the efficiency $\eta$ (see Eq. 1, Sect 2.3) in the bottom graph of Fig. 8, which has its minimum of $96.9 \%$ at the lowest stride frequency (i.e. $K_{\mathrm{h}}=0 \mathrm{Nm} / \mathrm{rad}$ ). The accompanying time series of one gait cycle of CPG-controlled walking in Fig 6a (black lines) shows that just before heel strike and just after toe-off, the moment of force $M_{\mathrm{r}}$ is indeed a short period of time out of 


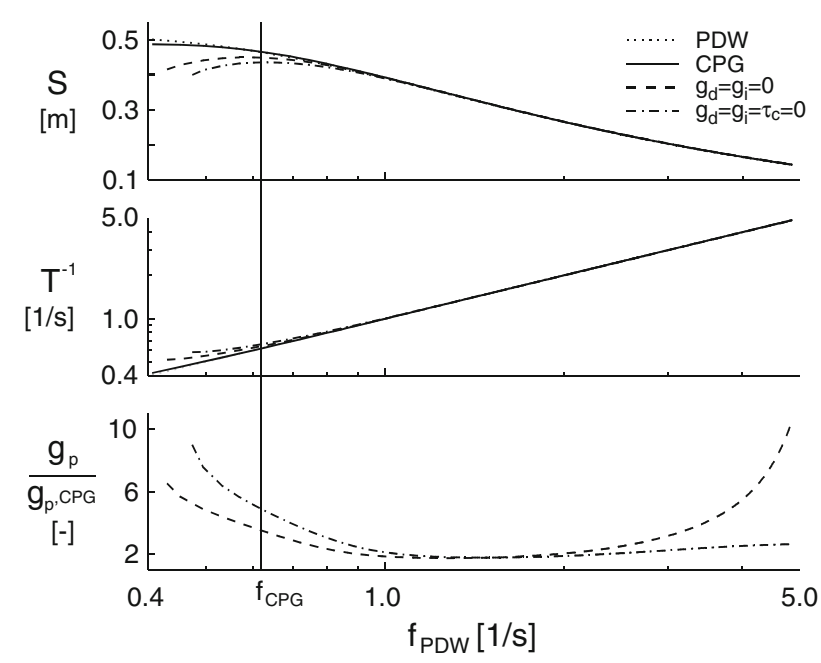

Fig. 7 Resonance tuning for same speed as passive dynamic walker (Fig. 5). The top graph shows the stride length $S$, the middle graph shows the stride frequency $1 / T$, and the bottoms graph shows the afferent strength necessary to maintain speed, relative to the default CPG

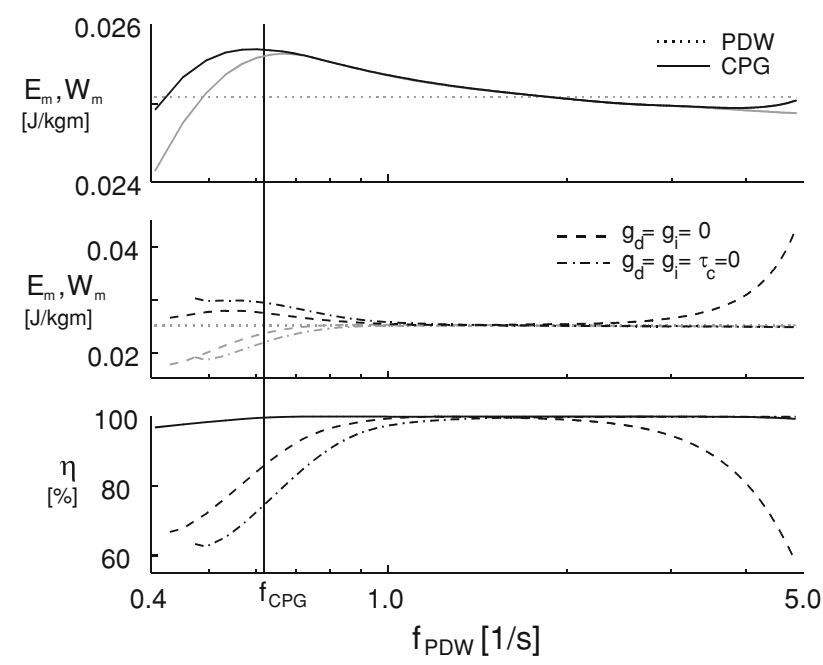

Fig. 8 Energy and work for the gait solutions shown in Fig. 7. The top graph shows the energy expenditure $E_{\mathrm{m}}$ of the muscles (solid black line) and their performed work $W_{\mathrm{m}}$ (solid grey line) versus the work $W_{\mathrm{g}}$ done by gravity in case of passive dynamic walking (dotted grey line). The middle graph shows $E_{\mathrm{m}}$ and $W_{\mathrm{m}}$ in case of no derivative and integral feedback to the half-center model (dashed black and grey lines, respectively) and in case of-besides the lack of integral and derivative feedback - the absence of time delay $\tau_{\mathrm{c}}$ (dashed-dot black and grey lines, respectively); both are plotted versus $W_{\mathrm{g}}$ (dotted grey line). The bottom graph shows the accompanying efficiency $\eta$ of the muscles in CPG-controlled walking (same legend applies as in top and middle graph). The endogenous frequency $f_{\mathrm{CPG}}$ is shown by a vertical line

phase with the angular velocity $\omega_{\mathrm{r}}$, thus performing negative work. Figure $6 \mathrm{~b}$ shows that for the highest considered stride frequency (i.e. $K_{\mathrm{h}}=61.05 \mathrm{Nm} / \mathrm{rad}$ ), $M_{\mathrm{r}}$ is only a very short period of time-just before heel strike—out of phase with $\omega_{\mathrm{r}}$, giving a $\eta$ of $99.4 \%$. The small differences in work $W_{\mathrm{m}}$ performed by the CPG-controlled muscles (solid grey line) and work $W_{\mathrm{g}}$ performed by gravity for passive dynamic walking (dotted grey line)—shown in the top graph of Fig. 8 - can be explained by small differences in the stride length $S$ and the swing leg's angular velocity at heel strike. At low stride frequencies, the foot mass looses more kinetic energy at heel strike in CPG-controlled walking than in passive dynamic walking (see zoom-in of $\omega_{\mathrm{r}}$ in Fig. 6a), while it is vice versa at high frequencies. This causes $W_{\mathrm{m}}$ to be smaller than $W_{\mathrm{g}}$ for high stride frequencies and higher than $W_{\mathrm{g}}$ for low frequencies, with the exception of the lowest frequencies. At the lowest stride frequencies the smaller stride length in CPG-controlled walking means smaller collision losses, which cause $W_{\mathrm{m}}$ to be smaller than $W_{\mathrm{g}}$.

It should be noted that the route to chaos, which is present in passive dynamic walking (Fig. 4), has not been encountered in CPG-controlled walking. This is based on a vast amount of simulations, many of which have not been discussed in this paper. The CPG seems to prevent or retard this route to chaos. Stable symmetric gait solutions exist for a certain parameter space and become unstable almost immediately after leaving this parameter space, sometimes preceded by asymmetric gait and one or two period doubling bifurcations. Thus, it could be that the route to chaos is present, but is compressed by the CPG into a very small area of the parameter space.

\subsubsection{The influence of the integral and derivative feedback}

Aforementioned results show that the CPG is able to control gait in an energy efficient way by resonance tuning. The type of afferent feedback to the half-center model is important in achieving this. To show the influence of integral and derivative feedback of the legs' angles to the half center model, we also performed simulations without these types of feedback. The dashed line in Fig. 7 shows the entrained gait solutions when there is no feedback of angular velocity or the integrated angle (i.e. $g_{\mathrm{d}}=g_{\mathrm{i}}=0$ ), again with the afferent strength adapted to match the passive dynamic walking velocities. The influence of integral feedback shows at low frequencies. Without it, the stride frequency $1 / T$ deviates substantially from $f_{\mathrm{PDW}}$ below $f_{\mathrm{CPG}}$. Walking without hip stiffness is no longer possible (the minimal $K_{\mathrm{h}}$ for stable gait is 0.05 ). Even at and beyond $f_{\mathrm{CPG}}$ small deviations are present. This poor resonance tuning behavior causes to drop the efficiency $\eta$ of the muscles to a minimum of $66.8 \%$ for the lowest stride length (bottom graph, Fig. 8). Although $W_{\mathrm{m}}$ is smaller than $W_{\mathrm{g}}$ at low frequencies-merely due to smaller stride lengths - the bad efficiency causes $E_{\mathrm{m}}$ to be larger than $W_{\mathrm{g}}$ (middle graph, Fig. 8). The influence of derivative feedback shows at high frequencies. Although without derivative feedback $1 / T$ is only slightly lower than $f_{\mathrm{PDW}}$ at high frequencies (top graph, Fig. 7), the efficiency $\eta$ goes down to $58.3 \%$ for the highest stride frequency. This shows that $\eta$ is a 
better measure for resonance tuning than the entrained stride frequency itself. By increasing the afferent strength significantly (bottom graph, Fig. 7), the entrained frequency comes close to $f_{\mathrm{PDW}}$, giving a false sense of good resonance tuning behavior, while in fact the energy expenditure is very high (middle graph, Fig. 8).

The dashed-dot lines in Fig. 7 and 8 show simulations for which-besides the integral and derivative afferent feedback - the time delay $\tau_{\mathrm{c}}$ in the loop coupling the CPGs to the limbs is absent (i.e. $g_{\mathrm{d}}=g_{\mathrm{i}}=\tau_{\mathrm{c}}=0$ ). These show almost perfect resonance tuning at high stride frequencies with a minimum efficiency $\eta$ of $99.97 \%$ at the highest stride frequency. Hence, these results clearly show that velocity feedback is necessary to compensate for the time delay in the loop, just as in rhythmic single limb movement (Verdaasdonk et al. 2006, 2007b). At low stride frequencies, the absence of time delay makes the resonance tuning worse with a minimum efficiency $\eta$ of $62.5 \%$ at the lowest stride frequency. Moreover, a minimum $K_{\mathrm{h}}$ of $0.15 \mathrm{Nm} / \mathrm{rad}$ is necessary to obtain stable gait. The performance at low stride frequencies is worse, because absence of time delay means absence of extra phase lag in the feedback loop to the half-center model. Extra phase lag is necessary for frequencies below the CPG's endogenous frequency to obtain resonance tuning (for a detailed explanation, see Verdaasdonk et al. (2006)). In fact, this is the reason that integral feedback is necessary for achieving energy efficient walking at low stride frequencies.

\subsubsection{Relationship between velocity feedback and time delay in the loop}

Figure 9 shows the gait solutions for increasing time delay in case there is no velocity feedback (i.e. $g_{\mathrm{d}}=0$ ), default velocity feedback (i.e. $g_{\mathrm{d}}=0.08 g_{\mathrm{p}}$ ) and velocity feedback for which the strength is linearly dependent on the size of the delay (i.e. $g_{\mathrm{d}}=0.08 g_{\mathrm{p}} \tau_{\mathrm{c}} / 50 \times 10^{-3}$ ). We start off with the gait solution of Figs. 7 and 8 belonging to a stride frequency $f_{\text {PDW }}$ of $2.01 / \mathrm{s}$ (i.e. $K_{\mathrm{h}}$ is $10 \mathrm{Nm} / \mathrm{rad}$ ) and leave the accompanying afferent positional gain $g_{\mathrm{p}}$ at the constant value of 19.0 for all simulations. The default velocity feedback (solid lines) shows that the value of $g_{\mathrm{d}}$ relative to $g_{\mathrm{p}}$ is laid out to compensate phase lags of time delays around $50 \mathrm{~ms}$. The efficiency $\eta$ is close to $100 \%$ and the gait velocity $v$ is close to that of passive dynamic walking as long as the time delay is close to $50 \mathrm{~ms}$. Much lower and much higher time delays will cause bad resonance tuning behavior, with accompanying bad efficiency and lower gait velocities. The energy expenditure in these cases is less, because the stride length $S$ diminishes with $v$, which means lower collision costs. No velocity feedback (dotted lines) causes the efficiency $\eta$ to be near $100 \%$ only in case of no delay. Even in that case the gait velocity $v$ is lower than in passive dynamic walking, because the afferent gain $g_{\mathrm{p}}$ is not adapted to match the latter veloc-

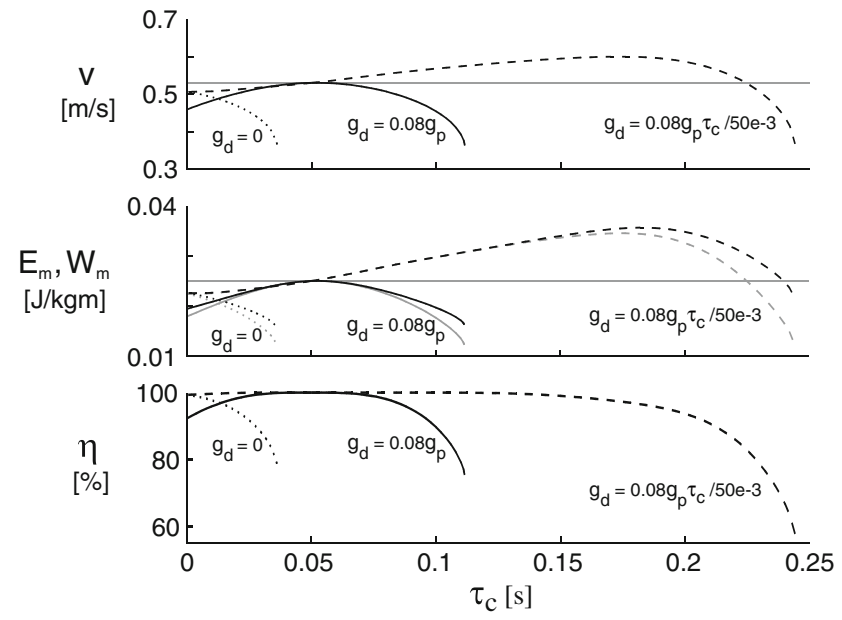

Fig. 9 Compensation of time delay by velocity feedback. The top graph shows the gait velocity $v$, the middle graph shows the muscles' energy expenditure $E_{\mathrm{m}}$ and their performed work $W_{\mathrm{m}}$ (black and grey lines, respectively), and the bottom graph shows the muscle efficiency $\eta$. The dotted lines represent gait solutions obtained without derivative feedback to the half-center model (i.e. $g_{\mathrm{d}}=0$ ), the solid lines represent gait solutions for the default velocity feedback (i.e. $g_{\mathrm{d}}=0.08 g_{\mathrm{p}}$ ), and the dashed lines represent gait solutions for velocity feedback with strength proportional to the time delay $\tau_{\mathrm{c}}$ in the loop (i.e. $\left.g_{\mathrm{d}}=0.08 g_{\mathrm{p}} \tau_{\mathrm{c}} / 50 \times 10^{-3}\right)$

ity. Hence, the CPG gets less total afferent input (as there is no velocity feedback) and will walk with the same stride frequency, but with lower stride length $S$ and therefore with less energy expenditure $E_{\mathrm{m}}$.

The combination of positional and velocity feedback is similar to a PD-controller and gives maximal $90^{\circ}$ phase lead. As the phase lag of the time delay increases with frequency $\left(H_{\tau}=\mathrm{e}^{-j \omega \tau}\right)$, there is a limit to the time delay, which can be compensated. The maximal time delay for which stable walking is possible is $0.244 \mathrm{~s}$ and is shown by the dashed lines in Fig. 9, which represent velocity feedback for which the strength is linearly dependent on the size of the delay. For this type of velocity feedback, the efficiency $\eta$ shows that very good resonance tuning behavior is achieved for time delays up to $0.15 \mathrm{~s}$. In other words, velocity feedback is essential for achieving stable and energy efficient walking when large time delays are present in the neural pathways.

\subsection{Velocity Control}

For a given hip stiffness $K_{\mathrm{h}}$, increasing gait velocity $v$ of the CPG-controlled walker is achieved by increasing the energy expenditure of the muscles. This can be accomplished by increasing the afferent gain $g_{\mathrm{p}}$, the efferent gain $k_{\mathrm{eE}}$, or the supra-spinal input $u_{0}$. Figure 10 shows the range of hip stiffness values $K_{\mathrm{h}}$ at which stable gait is possible for afferent strengths of 2, 20,60, and 100 . For a given hip stiffness $K_{\mathrm{h}}$, the velocity $v$ is increased by increasing $g_{\mathrm{p}}$ (top graph). The 

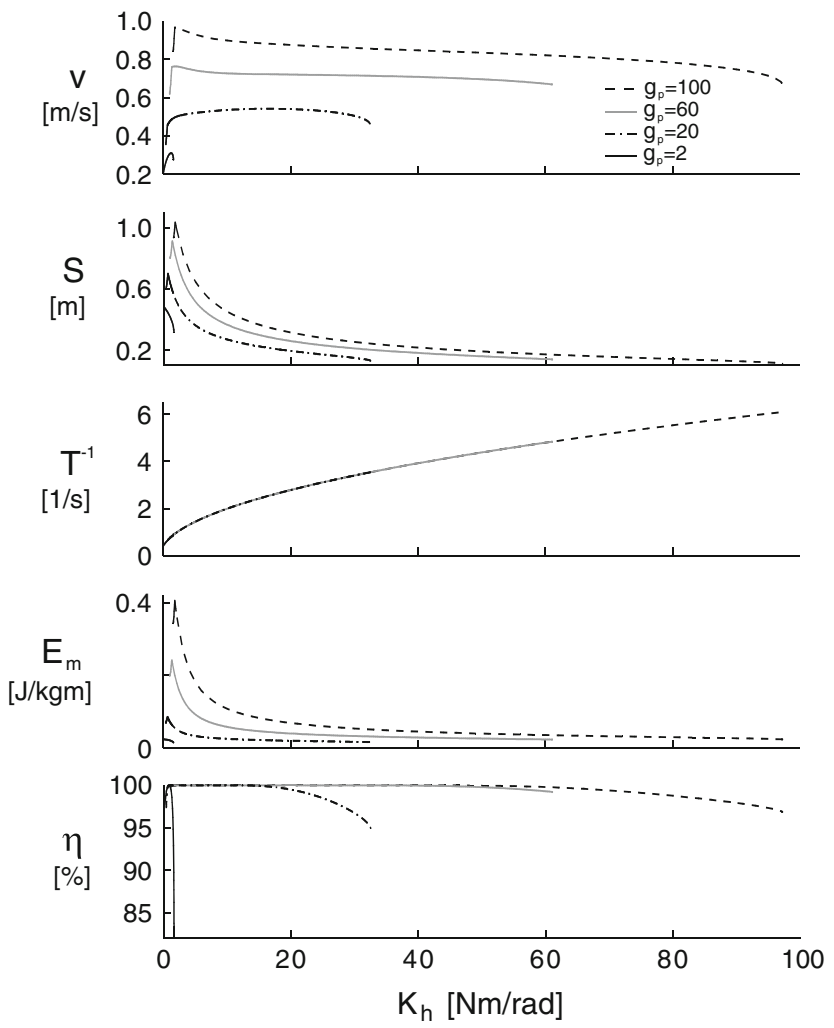

Fig. 10 Changing gait velocity by adaptation of afferent strength $g_{\mathrm{p}}$ for a range of hip stiffness values $K_{\mathrm{h}}$. Shown are from top to bottom graph: gait velocity $v$, stride length $S$, stride frequency $1 / T$, energy expenditure $E_{\mathrm{m}}$, and muscle efficiency $\eta$. The gait solutions are shown for an afferent strength $g_{\mathrm{p}}$ of 2 (solid black lines), 20 (dashed-dot black lines), 60 (solid grey lines), and 100 (dashed black lines)

increasing gait velocity is achieved by increasing the stride length $S$ (second graph), because the CPG tunes into the resonance frequency of the walker regardless of the afferent strength $g_{\mathrm{p}}$ (within certain limits), and is accompanied by a higher energy expenditure $E_{\mathrm{m}}$ (fourth graph). The fact that the CPG tunes into the walker's resonance frequency for all afferent strengths $g_{\mathrm{p}}$ is shown by the overlapping plots of the stride frequency $1 / T$ versus the hip stiffness $K_{\mathrm{h}}$ (middle graph). This good resonance tuning behavior is also shown by the muscle efficiency $\eta$ (bottom graph). The range of resonance frequencies (i.e. range of values of $K_{\mathrm{h}}$ ) for which stable walking is possible increases with increasing $g_{\mathrm{p}}$, although the lowest possible stride frequency becomes somewhat higher. For given afferent strength $g_{\mathrm{p}}$, higher hip stiffness $K_{\mathrm{h}}$ does not affect the gait velocity $v$ much (top graph), as long as $K_{\mathrm{h}}$ is in the range where resonance tuning behavior is almost perfect, i.e. $\eta$ close to $100 \%$ (bottom graph). However, the energy expenditure $E_{\mathrm{m}}$ is much less for higher $K_{\mathrm{h}}$, because of the lower collision costs associated with smaller stride lengths $S$.

Hence, the stride length $S$ is increased or decreased by increasing or decreasing the afferent gain $g_{\mathrm{p}}$, the efferent gain
$k_{\mathrm{eE}}$, or the supra-spinal input $u_{0}$, while the stride frequency $1 / T$ stays close to the mechanical resonance frequency of the walker due to the resonance tuning behavior of the CPG. This way, a range of gait velocities $v$ can be achieved by changing only one parameter. For example, for given $g_{\mathrm{p}}$ of 20 and $K_{\mathrm{h}}$ of 5 , a range of gait velocities is achieved from 0.28 up to $0.75 \mathrm{~m} / \mathrm{s}$ - with accompanying range of stride lengths from 0.20 to $0.54 \mathrm{~m}$ - by changing the supra-spinal input $u_{0}$ from 0.12 to 8.19 .

The range of velocities for which stable gait is possible can be made much larger by increasing and decreasing the walker's resonance frequency with gait velocity. The resonance frequency can be increased by increasing the hip stiffness $K_{\mathrm{h}}$ by a local positional feedback, just as a stretch reflex can increase the muscle stiffness (Verdaasdonk et al. 2004a) and thereby the resonance frequency of a limb (Verdaasdonk et al. 2007b). Thus, by making the local positional feedback gain dependent (e.g. proportionally) on $g_{\mathrm{p}}, k_{\mathrm{eE}}$, or $u_{0}$, a large range of gait velocities can be obtained by controlling only one parameter $\left(g_{\mathrm{p}}, k_{\mathrm{eE}}\right.$, or $\left.u_{0}\right)$. It is noted that controlling $K_{\mathrm{h}}$ in this way costs energy, as the obtained resonance frequency differs from the real mechanical resonance frequency. This additional energy expenditure is not taken into account in the presented results, since $K_{\mathrm{h}}$ is considered to be an energy conserving spring in this study.

\section{Discussion}

\subsection{Efficiency}

It is generally agreed that human walking is very energy efficient. Waters and Mulroy (1999) showed that the metabolic energy expenditure at (near) optimal walking speed (around $1.3 \mathrm{~m} / \mathrm{s}$ ) is about $3.1 \mathrm{~J} /(\mathrm{kg} \mathrm{m})$ for young adults. Taking into account that the metabolic efficiency of human muscular labor is about $30 \%$, this agrees with the inverse dynamics analysis of van der Kooij et al. 2003) that showed the positive mechanical work done by the joint muscles to be around $0.8 \mathrm{~J} /(\mathrm{kg} \mathrm{m})$.

A good comparison of energy expenditures of our CPG-controlled gait model with human walking is hard to make. On the one hand, our gait model has no knees and feet. If it had those, it could walk even more efficiently, especially at higher velocities (i.e. larger stride lengths). On the other hand, our model is a 2D model without a trunk, while humans need energy to maintain stability in a 3D environment (e.g. actively swinging the arms and balancing the trunk costs energy). If a comparison is to be made between the mechanical energy expenditure of our CPG-controlled gait model and human walking, our results show that our gait model is much more efficient at low speeds (expenditure is much less than $0.8 \mathrm{~J} /(\mathrm{kg} \mathrm{m}))$. However, if our gait model has low 
hip stiffness it is less efficient than human walking at the (near) optimal human walking velocity of $1.3 \mathrm{~m} / \mathrm{s}$ (you can see this by extrapolating Fig. 10), because high stride lengths are necessary at high velocities with the accompanying large kinetic energy losses at heel strike. However, with higher hip stiffness our gait model could walk more efficiently than humans.

It is more appropriate to compare the energy efficiency of our CPG-controlled gait model with passive dynamic walking for the same passive walker (i.e. same segment model). Passive dynamic walking is considered to be very efficient (Garcia et al. 1998, McGeer 1990), although it has a small basin of attraction (Schwab and Wisse 2001), that is, poor stability properties. Fig. 8 shows that the CPG achieves control of the passive walker with similar energy expenditure on flat ground compared to passive dynamic walking, while it can achieve speeds that greatly exceed the region of stability of passive dynamic walking.

Actually, the best way to determine the energy efficiency of muscle activated walking is by looking at the efficiency $\eta$ of the muscles. As shown by the simulations in this study (e.g. Fig. 8), the CPG achieves an efficiency $\eta$ of (nearly) $100 \%$ for large ranges in mechanical properties of the passive walker (e.g. the resonance frequency) without changing any of the CPG-related parameters in the model.

\subsection{Robustness versus efficiency}

In this study, we have focused on the energy efficiency of CPG-controlled walking. However, CPGs also play an important role in the recovery from small and medium-sized perturbations, and even from large perturbations, as was shown for a model of rhythmic arm movement in a previous study (Verdaasdonk et al. 2007b). In that study the forearm was also actuated at its resonance frequency by a CPG that was modeled in the same way as the one in this study. It showed that the CPG's contribution to the recovery of the forearm after large perturbations $(40 \mathrm{Nm}$ over an angular range of $0.1 \mathrm{rad}$ ) was quite high ( $41 \%$, the rest came from reflexes).

Although the CPG in the current study also adds a reasonable amount of robustness to the walking model, compared to uncontrolled passive dynamic walking, the question arises whether efficiency does not come at the expense of robustness. In a previous study we showed that there is indeed such a trade-off between energy consumption and robustness (Verdaasdonk et al. 2007a) in a similar CPG-controlled bipedal walking model (although that one could not passively walk down a slope, because it had human inertial distribution). The study showed that the robustness against large perturbations is increased with the velocity of the swing leg, which agrees with the statement of Wisse et al. (2005) that you will never fall forward if the swing leg is put fast enough in front of the stance leg. There are two methods to increase swing leg velocities and by that the robustness against large perturbations. In the current gait model with resonance tuning it is achieved by larger stride lengths. This will indeed cost more energy per unit distance walked, because of the mechanics of the passive dynamic walker: the change in direction of the hip mass' velocity at heel strike increases with stride length, which means more kinetic energy losses. Another method would be not to use resonance tuning, but to accelerate the swing leg at the beginning of the swing when the velocity is still in the reverse direction and to decelerate the leg before the end of the swing (i.e. break). This can be achieved by other feedback gains of the leg's sensory information to the CPG, but will cost a lot of energy because a lot of negative work will be performed by the muscles. The former method (i.e. resonance tuning) seems a better alternative, because for a more sophisticated passive dynamic walker with knees, feet and a way to store the kinetic energy now lost at heel strike (e.g. tendons) the kinetic energy losses can be minimized, while not actuating a passive dynamic walker in its natural dynamics (i.e. eigenmode) will always mean energy losses.

Besides increasing the robustness by increasing the swing leg velocity, which is mechanical in origin, it could be a good idea to extend the CPG or to add reflexes to the walking model in such a way that the walker is pulled back harder towards the limit cycle (i.e. the normal unperturbed gait cycle) if it is perturbed further away from it. This way, a larger robustness can be achieved, while it only costs more energy in case of actual perturbations. Thus, reflexes with non-linear gains-being larger when further away from the unperturbed gait cycle-could improve the robustness as well as the speed of recovery, while leaving the limit cycle of the rhythmic movement—and thus the corresponding energy efficiencyintact.

\subsection{Conclusion}

The most efficient way to actuate a 'mechanical oscillator'in this case a passive dynamic walker-is at its resonance frequency (i.e. resonance tuning). This study has shown that given the proper afferent feedback the CPG is able to automatically tune into this resonance frequency, thereby providing energy efficient gait control. Feedback of positional information provides resonance tuning above the endogenous frequency of the CPG. Integral feedback provides resonance tuning at and below the endogenous frequency. Feedback of velocity information is necessary to compensate for the time delay in the loop, coupling limb to CPG; without velocity feedback resonance tuning is not possible at high stride frequencies.

Considering the mechanical limitations of the passive dynamic walker (i.e. no feet, no knees, etc.), the gait velocity 
can be varied over quite a large range, while keeping the energy efficient behavior of passive dynamic walking intact. These velocity changes can be achieved by changing only a single parameter. Increasing the strength of one or more of the CPG inputs (afferent gain, efferent gain, or supra-spinal input) leads to a higher gait velocity. Since the CPG actuates the passive dynamic walker at its resonance frequency, the velocity change will be achieved by a change in stride length.

A change in stride frequency will only occur if the resonance frequency of the passive dynamic walker is changed, because the CPG will then actuate the passive dynamic walker at its new resonance frequency.

Our CPG model on the one hand might elucidate how humans achieve energy efficient walking, and on the other hand can be used as (part of a) gait controller in applications such as walking robots or powered walking orthoses.

Open Access This article is distributed under the terms of the Creative Commons Attribution Noncommercial License which permits any noncommercial use, distribution, and reproduction in any medium, provided the original author(s) and source are credited.

\section{Appendix A: Ground reaction forces}

The ground reaction force for a foot standing on the ground, $F_{\mathrm{Gjd}}$, is modeled by viscous damping in both $x$ - and $y$-direction and stiffness only in $y$-direction:

$F_{\mathrm{Gjx}}=-B_{\mathrm{x}} \dot{x}_{\mathrm{fj}}$

$F_{\mathrm{Gjy}}= \begin{cases}-B_{\mathrm{y}} \dot{y}_{\mathrm{fj}}-K_{\mathrm{y}} y_{\mathrm{fj}} & \text { if } \dot{y}_{\mathrm{fj}}<0 \\ -K_{\mathrm{y}} y_{\mathrm{fj}} & \text { else }\end{cases}$

with $B_{\mathrm{x}}, B_{\mathrm{y}}$ and $K_{\mathrm{y}}$ constant damping and stiffness factors, chosen such that an overdamped contact with negligible slip is obtained, $x_{\mathrm{jf}}$ and $y_{\mathrm{jf}}$ the co-ordinates of the feet relative to a base point on the ground. The indices $j$ and $d$ have the following meaning:

$j=\{r, l\} \quad$ leg index

$d=\{x, y\} \quad$ direction ground reaction force

Since our walker does not have knees, ground-clearance during swing phase must be provided artificially. This is accomplished by a ground reaction force $G_{\mathrm{fjd}}$ at foot $j$, stated as follows:

$G_{\mathrm{fjd}}= \begin{cases}F_{\mathrm{Gjd}} & \text { if leg } j \text { in Stance Phase } \\ 0 & \text { else }\end{cases}$

A leg is said to be in stance phase when its foot is below ground level and its angular velocity is below a threshold value $\omega_{\mathrm{G}}=0.1\left(y_{\mathrm{fj}}<0 \& \omega_{\mathrm{j}}<\omega_{\mathrm{G}}\right)$. The threshold value $\omega_{\mathrm{G}}$ is used because at very low speeds, heel strike (the beginning of stance phase) can occur at slightly positive angular velocity.

\section{Appendix B: Lagrange's equations for the passive dynamic walker}

The indices $j, n$, and $d$ in this appendix have the following meaning:

$j=\{r, l\}$

$n=\{1,2,3,4\} \quad$ generalized co-ordinate index

$d=\{\mathrm{x}, \mathrm{y}\} \quad$ direction ground reaction force

The generalized co-ordinates $\mathbf{q}$ are defined as the position of the hip mass $m_{h}$ in the global system, $\left(x_{\mathrm{h}}, y_{\mathrm{h}}\right)$, and the angles of the right and left leg relative to the vertical (i.e. segment angles), $\theta_{\mathrm{r}}$ and $\theta_{1}$ :

$\boldsymbol{q}=\left[\begin{array}{llll}x_{\mathrm{h}} & y_{\mathrm{h}} & \theta_{\mathrm{r}} \theta_{\mathrm{l}}\end{array}\right]^{\mathrm{T}}$

The positions of the feet $\mathbf{x}_{\mathbf{f}}=\left[x_{\mathrm{fr}} y_{\mathrm{fr}} x_{\mathrm{fl}} y_{\mathrm{ff}}\right]^{\mathrm{T}}$ are expressed in $\mathbf{q}$ as follows:

$$
\begin{aligned}
& x_{\mathrm{fj}}(\boldsymbol{q})=x_{\mathrm{h}}+l_{\text {leg }} \sin \left(\theta_{\mathrm{j}}\right) \\
& y_{\mathrm{fj}}(\boldsymbol{q})=y_{\mathrm{h}}-l_{\text {leg }} \cos \left(\theta_{\mathrm{j}}\right)
\end{aligned}
$$

The scalar kinetic energy function $T(\mathbf{q})$ is given by:

$$
\begin{aligned}
T(\boldsymbol{q}, \dot{\boldsymbol{q}})= & \frac{1}{2} m_{\mathrm{h}} \dot{x}_{\mathrm{h}}^{2}+\frac{1}{2} m_{\mathrm{h}} \dot{y}_{\mathrm{h}}^{2} \\
& +\sum_{\mathrm{j}}\left(\frac{1}{2} m_{\mathrm{f}} \dot{x}_{\mathrm{fj}}^{2}(\boldsymbol{q})+\frac{1}{2} m_{\mathrm{f}} \dot{y}_{\mathrm{fj}}^{2}(\boldsymbol{q})\right)
\end{aligned}
$$

The scalar gravitational potential energy function $\mathrm{V}_{\mathrm{g}}(\mathbf{q})$ is given by:

$$
\begin{aligned}
& V(\boldsymbol{q})=m_{\mathrm{h}} g\left(\sin (-\alpha) x_{\mathrm{h}}+\cos (-\alpha) y_{\mathrm{h}}\right)+\sum_{\mathrm{j}} \frac{1}{2} K_{\mathrm{h}} \theta_{\mathrm{j}}^{2} \\
& +\sum_{\mathrm{j}} m_{\mathrm{f}} g\left(\sin (-\alpha) x_{\mathrm{fj}}(\boldsymbol{q})+\cos (-\alpha) y_{\mathrm{fj}}(\boldsymbol{q})\right)
\end{aligned}
$$

The foot-ground contact forces Eq. A.2 are written as vector $\boldsymbol{G}_{f}\left(\boldsymbol{x}_{f}\right)=\left[G_{\mathrm{frx}} G_{\mathrm{fry}} G_{\mathrm{flx}} G_{\mathrm{fly}}\right]^{\mathrm{T}}$, which is expressed in local foot co-ordinates $\boldsymbol{x}_{\mathbf{f}}$ and must be transformed to an expression in generalized co-ordinates before entering Lagrange's equations of motion:

$\mathbf{G}_{\mathrm{n}}(\boldsymbol{q}, \dot{\boldsymbol{q}})=\sum_{\mathrm{k}=1}^{4} \boldsymbol{G}_{\boldsymbol{f k}}\left(\boldsymbol{x}_{f}\right) \frac{\partial \boldsymbol{x}_{\boldsymbol{f} \mathrm{k}}}{\partial q_{\mathrm{n}}}$

Lagrange's equations of motion are now:

$\frac{\mathrm{d}}{\mathrm{d} t}\left(\frac{\partial T}{\partial \dot{q}_{\mathrm{n}}}\right)-\frac{\partial T}{\partial q_{\mathrm{n}}}+\frac{\partial V}{\partial q_{\mathrm{n}}}=\mathbf{M}_{\mathrm{n}}+\mathbf{G}_{\mathrm{n}}$

with $\mathbf{M}$ the vector of generalized forces, comprising the CPG-controlled hip moments of force (Eq. C.8): $\mathbf{M}=\left[\begin{array}{llll}0 & 0 & \mathbf{M}_{\mathrm{r}} \mathbf{M}_{1}\end{array}\right]^{\mathrm{T}}$.

\section{Parameters}

$m_{\mathrm{h}}=1.0 \mathrm{~kg}$, hip mass

$m_{\mathrm{f}}=0.04 \mathrm{~kg}$, foot mass

$l_{\text {leg }}=1.0 \mathrm{~m}$, leg length 
$g=9.81 \mathrm{~m} / \mathrm{s}^{2}$, gravity constant

$K_{\mathrm{y}}=1 \times 10^{5} \mathrm{~N} / \mathrm{m}$, ground stiffness in $y$-direction

$B_{\mathrm{y}}=1 \times 10^{4} \mathrm{Ns} / \mathrm{m}$, ground damping in $y$-direction

$B_{\mathrm{x}}=1 \times 10^{6} \mathrm{Ns} / \mathrm{m}$, ground damping in $x$-direction

\section{Variables with default values}

$\alpha=2.6 \times 10^{-3} \mathrm{rad}$, ground slope

$K_{\mathrm{h}}=0 \mathrm{Nm} / \mathrm{rad}$, rotational stiffness of the hip

\section{Appendix C: Central pattern generator}

The dynamics for the CPG coupled to leg $j(j=\{r, l\})$ are given by the following equations:

$$
\begin{aligned}
& \dot{u}_{\mathrm{Fj}}=\frac{1}{\tau_{\mathrm{r}}}\left(u_{0}-u_{\mathrm{Fj}}-\beta v_{\mathrm{Fj}}-w y_{\mathrm{Ej}}-g_{\mathrm{p}} \theta_{\mathrm{j}}\left(t-\tau_{\mathrm{c}}\right)\right. \\
& \left.\quad-g_{\mathrm{d}} \omega_{\mathrm{j}}\left(t-\tau_{\mathrm{c}}\right)-g_{\mathrm{i}} \varphi_{\mathrm{j}}\left(t-\tau_{\mathrm{c}}\right)\right) \\
& \dot{v}_{\mathrm{Fj}}=\frac{1}{\tau_{\mathrm{a}}}\left(y_{\mathrm{Fj}}-v_{\mathrm{Fj}}\right) \\
& \dot{u}_{\mathrm{Ej}}=\frac{1}{\tau_{\mathrm{r}}}\left(u_{0}-u_{\mathrm{Ej}}-\beta v_{\mathrm{Ej}}-w y_{\mathrm{Fj}}+g_{\mathrm{p}} \theta_{\mathrm{j}}\left(t-\tau_{\mathrm{c}}\right)\right. \\
& \left.\quad+g_{\mathrm{d}} \omega_{\mathrm{j}}\left(t-\tau_{\mathrm{c}}\right)+g_{\mathrm{i}} \varphi_{\mathrm{j}}\left(t-\tau_{\mathrm{c}}\right)\right) \\
& \dot{v}_{\mathrm{Ej}}=\frac{1}{\tau_{\mathrm{a}}}\left(y_{\mathrm{Ej}}-v_{\mathrm{Ej}}\right) \\
& \dot{\varphi}_{\mathrm{j}}=\frac{1}{\tau_{\mathrm{i}}}\left(\tau_{\mathrm{i}} \theta_{\mathrm{j}}-\varphi_{\mathrm{j}}\right)
\end{aligned}
$$

in which $u_{\mathrm{Fj}}, v_{\mathrm{Fj}}, u_{\mathrm{Ej}}$ and $v_{\mathrm{Ej}}$ are the state variables of the flexor $(F)$ and extensor $(E)$ centers and $\phi_{\mathrm{j}}$ is the integral of the angle $\theta_{\mathrm{j}}$. Integration of the angle $\theta_{\mathrm{j}}$ is assumed to be performed by an internal process of the CPG, which is modeled as leaking integrator (pole at $-1 / \tau_{\mathrm{i}}$ see Eq. C.5).

The outputs of the flexor and extensor centers, $y_{\mathrm{Fj}}$ and $y_{\mathrm{Ej}}$, are given by:

$$
\begin{aligned}
& y_{\mathrm{Fj}}=\max \left(0, u_{\mathrm{Fj}}\right) \\
& y_{\mathrm{Ej}}=\max \left(0, u_{\mathrm{Ej}}\right)
\end{aligned}
$$

The actuation moments of force of our CPG-controlled hip muscles are then given by:

$M_{\mathrm{j}}=k_{\mathrm{eE}} y_{\mathrm{Ej}}-k_{\mathrm{eF}} y_{\mathrm{Fj}}$

\section{Parameters}

$\tau_{\mathrm{r}}=0.18 \mathrm{~s}$, rise time constant

$\tau_{\mathrm{a}}=0.36 \mathrm{~s}$, adaptation time constant

$\tau_{\mathrm{i}}=10.0 \mathrm{~s}$, time constant for leaking integrator

$\beta=2.0$, strength adaptation effect

$w=2.0$, strength of reciprocal inhibition

$k_{\mathrm{eE}}=0.04$, efferent gain associated with extensor center

$k_{\mathrm{eF}}=0.1 k_{\mathrm{eE}}$, efferent gain associated with flexor center

\section{Variables with default values}

$u_{0}=1.0$, tonic input from supra-spinal centers

$g_{\mathrm{p}}=2.0$, feedback strength of limb angle

$g_{\mathrm{d}}=0.08 g_{\mathrm{p}}$, feedback strength of limb's angular velocity

$g_{\mathrm{i}}=2 \pi f_{\mathrm{CPG}} g_{\mathrm{p}}$, feedback strength of integrated limb angle

$\tau_{\mathrm{c}}=50 \times 10^{-3} \mathrm{~s}$, time delay in feedback loop

\section{References}

Amemiya M, Yamaguchi T (1984) Fictive locomotion of the forelimb evoked by stimulation of the mesencephalic locomotor region in the decerebrate cat. Neurosci Lett 50:91-96

Barbeau H, McCrea DA, O’Donovan MJ, Rossignol S, Grill WM, Lemay MA (1999) Tapping into spinal circuits to restore motor function. Brain Res Brain Res Rev 30:27-51

Borzova E, Hurmuzlu Y (2004) Passively walking five-link robot. Automatica 40:621-629

Burke RE (2001) The central pattern generator for locomotion in mammals. Adv Neurol 87:11-24

Cazalets JR, Borde M, Clarac F (1995) Localization and organization of the central pattern generator for hindlimb locomotion in newborn rat. J Neurosci 15:4943-4951

Dimitrijevic MR, Gerasimenko Y, Pinter MM (1998) Evidence for a spinal central pattern generator in humans. Ann NY Acad Sci 860:360-376

Donelan JM, Kram R, Kuo AD (2002) Mechanical work for step-tostep transitions is a major determinant of the metabolic cost of human walking. J Exp Biol 205:3717-3727

Feigenbaum MJ (1978) Quantitative universality for a class of non-linear transformations. J Stat Phys 19:25-52

Garcia M, Chatterjee A, Ruina A, Coleman M (1998) The simplest walking model: stability, complexity, and scaling. J Biomech Eng 120:281-288

Goswami A, Espiau B, Keramane A (1997) Limit cycles in a passive compass gait biped and passivity-mimicking control laws. Auton Robots 4:273-286

Goswami A, Thuilot B, Espiau B (1998) A study of the passive gait of a compass-like biped robot: Symmetry and chaos. Int J Rob Res 17:1282-1301

Grillner S, Ekeberg O, El Manira A, Lansner A, Parker D, Tegner J, Wallen P (1998) Intrinsic function of a neuronal network-a vertebrate central pattern generator. Brain Res Brain Res Rev 26:184197

Grillner S, McClellan A, Perret C (1981) Entrainment of the spinal pattern generators for swimming by mechano- sensitive elements in the lamprey spinal cord in vitro. Brain Res 217:380-386

Inman V, Ralston H, Todd F (1981) Human Walking. Williams and Wilkins, Baltimore

Koopman HFJM (1989) The three-dimensional analysis and prediction of human walking. Department of Mechanical Engineering. University of Twente, Enschede, The Netherlands

Kuo AD (2002) Energetics of actively powered locomotion using the simplest walking model. J Biomech Eng 124:113-120

MacKay-Lyons M (2002) Central pattern generation of locomotion: a review of the evidence. Phys Ther 82:69-83

Matsuoka K (1985) Sustained oscillations generated by mutually inhibiting neurons with adaptation. Biol Cybern 52:367-376

Matsuoka K (1987) Mechanisms of frequency and pattern control in the neural rhythm generators. Biol Cybern 56:345-353

McCrea DA (2001) Spinal circuitry of sensorimotor control of locomotion. J Physiol 533:41-50

McGeer T (1990) Passive dynamic walking. Int J Rob Res 9:62-82 
McMahon TA (1984) Muscles, reflexes, and locomotion. Princeton University Press, Princeton, pp 331

Mochon S, McMahon TA (1980) Ballistic walking. J Biomech 13:4957

Nishimaru H, Kudo N (2000) Formation of the central pattern generator for locomotion in the rat and mouse. Brain Res Bull 53:661-669

Schwab AL, Wisse M (2001) Basin of attraction of the simplest walking model. In: Proceedings of ASME 2001 design engineering technical conferences and computers and information in engineering conference, Pittsburgh, Pennsylvania

Shik ML, Severin FV, Orlovskii GN (1966) Control of walking and running by means of electrical stimulation of the mid-brain. Biophysics 11:756-765

Sqalli-Houssaini Y, Cazalets JR, Clarac F (1993) Oscillatory properties of the central pattern generator for locomotion in neonatal rats. $\mathrm{J}$ Neurophysiol 70:803-813

Taga G (1995a) A model of the neuro-musculo-skeletal system for human locomotion. I. Emergence of basic gait. Biol Cybern 73:97111

Taga G (1995b) A model of the neuro-musculo-skeletal system for human locomotion. II Real-time adaptability under various constraints. Biol Cybern 73:113-121

Taga G, Yamaguchi Y, Shimizu H (1991) Self-organized control of bipedal locomotion by neural oscillators in unpredictable environment. Biol Cybern 65:147-159

Van de Crommert HW, Mulder T, Duysens J (1998) Neural control of locomotion: sensory control of the central pattern generator and its relation to treadmill training. Gait Posture 7:251-263

van der Kooij H, Jacobs R, Koopman B, van der Helm F (2003) An alternative approach to synthesizing bipedal walking. Biol Cybern $88: 46-59$ van der Linde RQ (1999) Passive bipedal walking with phasic muscle contraction. Biol Cybern 81:227-237

Verdaasdonk BW, Koopman HF, Helm FC (2006) Energy efficient and robust rhythmic limb movement by central pattern generators. Neural Netw 19:388-400

Verdaasdonk BW, Koopman HF, Van Der Helm FC (2007a) Achieving energy efficient and robust bipedal gait with a CPG-controlled bipedal walker: Tuning the neural coupling gains. In: Williams TO (ed) Biological Cybernetics Research Trends. Nova Science, Hauppauge

Verdaasdonk BW, Koopman HF, Van der Helm FC (2007b) Resonance tuning in a neuro-musculo-skeletal model of the forearm. Biol Cybern 96:165-180

Verdaasdonk BW, Koopman HF, Van Gils SA, Van Der Helm FC (2004a) Bifurcation and stability analysis in musculoskeletal systems: a study in human stance. Biol Cybern 91:48-62

Verdaasdonk BW, Koopman HFJM, Van Gils SA, Van Der Helm FC (2004b) Stable walking with central pattern generators. In: Proceedings of the European Society of Biomechanics Congress, Eindhoven University of Technology, 's-Hertogenbosch, The Netherlands

Waters RL, Mulroy S (1999) The energy expenditure of normal and pathologic gait. Gait Posture 9:207-231

Whelan PJ (1996) Control of locomotion in the decerebrate cat. Prog Neurobiol 49:481-515

Wisse M, Schwab AL, van der Linde RQ, van der Helm FCT (2005) How to keep from falling forward: elementary swing leg action for passive dynamic walkers. Robotics, IEEE Transactions on [see also Robotics and Automation, IEEE Transactions on] 21:393-401 